\title{
Caracterização de sedimentos em viveiros de piscicultura na Amazônia Oriental,
}

\section{Brasil}

Characterization of sediments in fish farming ponds in the Eastern Amazon, Brazil

Caracterización de sedimentos en estanques de piscicultura en la Amazonía oriental, Brasil

Recebido: 08/01/2021 | Revisado: 12/01/2021 | Aceito: 18/01/2021 | Publicado: 21/01/2021

Raimundo Aderson Lobão de Souza

ORCID: https://orcid.org/0000-0003-0424-5008

Universidade Federal Rural da Amazônia, Brasil E-mail: adersonlobao@globo.com

Rodrigo Takata

ORCID: https://orcid.org/0000-0003-3385-6895 Fundação Instituto de Pesca do Estado do Rio de Janeiro, Brasil E-mail: takatarodrigo@gmail.com

Alex da Silva Lobão de Souza

ORCID: https://orcid.org/0000-0001-8136-9659 Instituto Federal de Educação, Ciência e Tecnologia do Pará, Brasil E-mail: alex.lobao@ifpa.edu.br

Mário Lopes da Silva Júnior

ORCID: https://orcid.org/0000-0001-9772-1290

Universidade Federal Rural da Amazônia, Brasil E-mail: mario.silva_junior@yahoo.com.br

Fabricio Nilo Lima da Silva

ORCID: https://orcid.org/0000-0002-6402-0540

Instituto Federal de Educação, Ciência e Tecnologia do Pará, Brasil E-mail: fabricio.nilo@ifpa.edu.br

\begin{abstract}
Resumo
O objetivo do estudo foi caracterizar o sedimento de viveiros com peixes e sem peixes da várzea estuarina da Amazônia Oriental, Pará, Brasil, após a calagem e adubação. Foi utilizado tambaquis (Colossoma macropomum), com comprimento total médio de $3,19 \mathrm{~cm}$ e peso total médio de $0,28 \mathrm{~g}$. Os tratamentos foram viveiros de várzea estuarina que receberam calagem, adubação e peixe (T1); viveiros com calagem e sem peixe (T2); e, viveiros sem qualquer preparação e com peixe (T3). A calagem para assepsia e correção de $\mathrm{pH}$, foi realizada com cal hidratada magnesiana e PRNT 90\% na quantidade de $1000 \mathrm{~kg} \mathrm{ha}^{-1}$. Além disso, efetuada a fertilização com esterco orgânico (esterco curtido de aves) na quantidade de $1000 \mathrm{~kg} \mathrm{ha}^{-1}$, após a aplicação do calcário. $\mathrm{O} \mathrm{pH}$ médio foi significativamente maior nos sedimentos (T1), seguido dos tratamentos T2 e T3, respectivamente. O carbono orgânico, $\mathrm{Mg}^{+2} \mathrm{e} \mathrm{Al}^{+3}$ trocáveis foram superiores $(p<0,05)$ em sedimentos de $T 1$, em comparação com T2 e T3, que apresentaram valores intermédios e inferiores $(\mathrm{p}<0,05)$, respectivamente. A quantidade de matéria orgânica e K trocável foram maiores $(\mathrm{p}<0,05)$ em T1 e T3 quando comparado ao T2. O P disponível e Ca trocável, foram significativamente maiores nos solos sem preparo calagem (T3) e inferiores ( $\mathrm{p}<0,05)$ nos tratamentos que receberam calagem (T1 e T2). O H ${ }^{+} \mathrm{Al}$ trocável foi maior $(\mathrm{p}<0,05)$ em T3, seguida por T1 e T2, respectivamente. Portanto, conclui-se que os solos de viveiros de piscicultura em várzeas na Amazônia são susceptíveis à calagem, e manejo de fertilizantes. O manejo correto na preparação dos viveiros em várzeas estuarinas pode melhorar a qualidade do solo para piscicultura neste tipo de ambiente.
\end{abstract}

Palavras-chave: Aquicultura; Colossoma macropomum; Solo.

\begin{abstract}
The objective of the study was to characterize the pond sediment with fish and without fish from the estuarine lowland of the Eastern Amazon, Pará, Brazil, after liming and fertilizing. Tambaquis (Colossoma macropomum) were used, with an average total length of $3.19 \mathrm{~cm}$ and an average total weight of $0.28 \mathrm{~g}$. The treatments were estuarine lowland ponds that received liming, fertilization and fish (T1); nurseries with liming and without fish (T2); and, nurseries without any preparation and with fish (T3). Liming for asepsis and $\mathrm{pH}$ correction was carried out with hydrated magnesium lime and PRNT 90\% in the amount of $1000 \mathrm{~kg} \mathrm{ha}^{-1}$. In addition, fertilization with organic manure (tanned manure from birds) in the amount of $1000 \mathrm{~kg} \mathrm{ha}^{-1}$, after. The average $\mathrm{pH}$ was significantly higher in sediments (T1), followed by treatments $\mathrm{T} 2$ and $\mathrm{T} 3$, respectively. Exchangeable organic carbon, $\mathrm{Mg}^{+2}$ and $\mathrm{Al}^{+3}$ were higher $(\mathrm{p}<0.05)$ in $\mathrm{T} 1$ sediments, compared to T2 and T3, which presented intermediate and lower values ( $\mathrm{p}<0.05$ ), respectively. The amount of organic matter and exchangeable $\mathrm{K}$ were higher $(\mathrm{p}<0.05)$ in $\mathrm{T} 1$ and $\mathrm{T} 3$ when compared to T2. The available $\mathrm{P}$ and exchangeable Ca were significantly higher in soils without liming preparation (T3) and lower $(\mathrm{p}<0.05)$ in treatments that received liming ( $\mathrm{T} 1$ and $\mathrm{T} 2)$. The exchangeable $\mathrm{H}^{+} \mathrm{Al}$ was higher $(\mathrm{p}<0.05)$ in $\mathrm{T} 3$, followed by $\mathrm{T} 1$
\end{abstract}


and T2, respectively. Therefore, it is concluded that the soils of fish farming nurseries in lowlands in the Amazon are susceptible to liming and fertilizer management. Correct management in the preparation of nurseries in estuarine floodplains can improve the quality of the soil for fish farming in this type of environment.

Keywords: Aquaculture; Colossoma macropomum; Ground.

\section{Resumen}

El objetivo del estudio fue caracterizar el sedimento del estanque con peces y sin peces de las tierras bajas estuarinas de la Amazonia Oriental, Pará, Brasil, después de encalar y fertilizar. Se utilizaron tambaquis (Colossoma macropomum), con una longitud total promedio de $3.19 \mathrm{~cm}$ y un peso total promedio de $0.28 \mathrm{~g}$. Los tratamientos fueron estanques estuarinos de tierras bajas que recibieron encalado, fertilización y peces (T1); viveros con encalado y sin peces (T2); y, viveros sin preparación y con pescado (T3). El encalado para asepsia y corrección de pH se realizó con cal magnésica hidratada y PRNT $90 \%$ en la cantidad de $1000 \mathrm{~kg} \mathrm{ha}^{-1}$. Además, la fertilización con estiércol orgánico (estiércol curtido de aves) en la cantidad de $1000 \mathrm{~kg} \mathrm{ha}^{-1}$, después. El pH promedio fue significativamente mayor en sedimentos (T1), seguido de los tratamientos T2 y T3, respectivamente. El carbono orgánico intercambiable, $\mathrm{Mg}^{+2}$ y Al$^{+3}$ fueron mayores $(\mathrm{p}<0.05)$ en los sedimentos $\mathrm{T} 1$, en comparación con $\mathrm{T} 2$ y $\mathrm{T} 3$, los cuales presentaron valores intermedios y menores $(\mathrm{p}<0.05)$, respectivamente. La cantidad de materia orgánica y $\mathrm{K}$ intercambiable fue mayor $(\mathrm{p}<0.05)$ en T1 y T3 en comparación con T2. El P disponible y el Ca intercambiable fueron significativamente mayores en suelos sin preparación de encalado $(\mathrm{T} 3)$ y menores $(\mathrm{p}<0.05)$ en tratamientos que recibieron encalado (T1 y T2). El intercambiable $\mathrm{H}+\mathrm{Al}$ fue mayor $(\mathrm{p}<0.05)$ en $\mathrm{T} 3$, seguido de $\mathrm{T} 1$ y T2, respectivamente. Por tanto, se concluye que los suelos de los viveros de piscicultura en tierras bajas de la Amazonía son susceptibles al encalado y manejo de fertilizantes. Una correcta gestión en la preparación de viveros en llanuras aluviales estuarinas puede mejorar la calidad del suelo para la piscicultura en este tipo de entornos.

Palabras clave: Acuicultura; Colosoma macropomum; Suelo.

\section{Introdução}

A condição do solo na aquicultura é tão importante quanto a qualidade da água, considerando a estrutura e função dos viveiros (Ostrensky \& Boeger, 1998; Prihutomo et al., 2016). O solo é um fator importante na aquicultura porque os viveiros são produzidos de material do solo e a condição do fundo destas estruturas influencia na qualidade da água e consequente na produção. Desta forma, correções das propriedades do solo, principalmente as químicas, são necessárias para melhorar as condições dos viveiros para a criação de organismos aquáticos (Ostrensky \& Boeger, 1998).

Muitas vezes a baixa qualidade do solo passa a ser um entrave, quando os aquicultores não conseguem explicar o baixo crescimento ou a queda na sobrevivência dos organismos aquáticos criados devido a problemas de doenças e parasitas, má qualidade dos alimentos, condições climáticas, qualidade da água prejudicada, manejo inadequado ou outras causas comuns (Boyd, 1982; 1990; Peterson, 1999, 2000; Queiroz et al., 2004). Além disso, no sedimento dos viveiros podem ocorrer bioacumulação química (Schmidt et al., 2000; Chelossi et al., 2003; Burton \& Landrum, 2004; Arauco et al., 2005; Lu et al., 2009; Chen et al., 2014) e a aplicação contínua desses produtos químicos pode promover o desenvolvimento e crescimento de bactérias resistentes e causar risco potencial de contaminação ao consumidor final (Grigorakis \& Rigos, 2011; Rico et al., 2012).

A camada superior de cinco centímetros do sedimento do fundo dos viveiro é a responsável pela qualidade da água nestes ambientes (Queiroz \& Boeira, 2006). Em geral, a deterioração da água está relacionada a um declínio na qualidade do fundo do viveiro, como resultado do acúmulo de sedimentos. Uma quantidade expressiva da matéria orgânica é removida dos viveiros através de uma rica decomposição da comunidade microbiana, e o nitrogênio é perdido por meio da volatilização e desnitrificação da amônia (Meijer \& Avnimelech, 1999; Gross et al., 1999; 2000). O restante da matéria orgânica, nitrogênio e fósforo proveniente de dietas formuladas será retida no sedimento e sujeito à atividade bacteriana (Boyd, 1995; Ostrensky; Boeger, 1998). Os organismos aquáticos retêm 13\% de carbono, $29 \%$ de nitrogênio e $16 \%$ de fósforo da dieta, e o restante é acumulado no fundo dos viveiros (Avnimelech \& Ritvo, 2003). Essa deposição de nutrientes no fundo do viveiro, originada de restos de materiais usados na dieta dos peixes, provoca alterações significativas nas propriedades químicas do solo, causando muitas vezes problemas de contaminação da água. 
A troca de cátions ou ânions depende principalmente da concentração do tipo de argila mineral, da quantidade de matéria orgânica, do grau de sua composição, além do pH do solo. Os cátions permutáveis são aqueles que podem ser trocados por cátions de uma solução salina adicionada. Em muitos casos, a definição de um cátion trocável é inteiramente direta, isto é, qualquer cátion adicionado trocará o cátion do solo (Thomas, 1982). Os cátions trocáveis $\mathrm{Ca}^{2+}, \mathrm{Mg}^{2+}$ e $\mathrm{K}^{+}$em solos estão relacionados com a disponibilidade de nitrogênio, perturbando a capacidade de sedimentação do tampão (Tian \& Niu, 2015). Em outras palavras, o equilíbrio entre o manejo alimentar e a deposição de nutrientes sobre o sedimento dos viveiros é necessário para incrementar a produção.

O objetivo do estudo foi caracterizar o sedimento de viveiros com peixes e sem peixes da várzea estuarina da Amazônia Oriental, Pará, Brasil, após a calagem e adubação.

\section{Material e Métodos}

O estudo foi desenvolvido na Unidade de Piscicultura Experimental de Várzea, da Universidade Federal Rural da Amazônia (UFRA), Campus de Belém. A Unidade está localizada em paralelo 01²7'20" S e meridiano 48³0'15". O Gr Segundo Koppen, classifica o clima regional como Afi, com chuvas durante todo o ano, com intenso período de chuvas entre dezembro e maio e chuvas menos intensas, entre junho e novembro. A temperatura média anual é de $25,9^{\circ} \mathrm{C}$, precipitação de $2.761 \mathrm{~mm}$ ano-1, umidade relativa de $86 \%$ e insolação de 2.389 horas ano-1.

Para o experimento, utilizou-se alevinos de tambaqui, Colossoma macropomum com comprimento total médio de 3,19 cm e peso total médio de 0,28 g. O desenho experimental foi em delineamento inteiramente casualizado (DIC). Os tratamentos foram viveiros de várzea estuarina que receberam calagem, adubação e peixe (T1); viveiros com calagem e sem peixe (T2); e, viveiros sem qualquer preparação e com peixe (T3). A calagem em T1 e T2, para assepsia e correção de pH, foi realizada com cal hidratada magnesiana e PRNT 90\% na quantidade de $1000 \mathrm{~kg} \mathrm{ha}^{-1}$. Além disso, efetuada a fertilização com esterco orgânico (esterco curtido de aves) na quantidade de $1000 \mathrm{~kg} \mathrm{~h}^{\mathrm{a}-1}$ foi realizada em T1 três dias após a aplicação do calcário.

As amostras de solo dos viveiros foram coletadas a uma profundidade de $30 \mathrm{~cm}$ abaixo da superfície, em intervalos de 10 dias, totalizando cinco amostras por viveiro, durante um período de 40 dias. As amostragens foram realizadas com coletor de PVC em modelo "S" e, após homogeneização, acondicionadas em sacos plásticos, devidamente etiquetadas e transportadas para o Laboratório de Química do Solo da UFRA, para análise. As amostras foram secas e peneiradas em malha de 2 mm e preparadas de acordo com a metodologia de Silva (1991).

As metodologias de análise dos elementos $\mathrm{Ca}^{+2}, \mathrm{Mg}^{+2}, \mathrm{Al}^{+3}, \mathrm{P}$ e K $\mathrm{K}^{+}$foram baseadas em Jackson (1962), Slavin (1968), Vetori (1969), Guimarães et al. (1970), Vogel (1981), Silva (1991) e EMBRAPA (1997a, 1997b). Os teores trocáveis de Ca, $\mathrm{Mg}$ e $\mathrm{Al}$ foram extraídos com $1 \mathrm{~mol}$ de $\mathrm{KCl} \mathrm{L}^{-1}$; Ca e $\mathrm{Mg}$ foram determinados por titulações com 0,0125 mol de EDTA L ${ }^{-1}$, o Al foi determinado por titulação com $0,025 \mathrm{~mol} \mathrm{de} \mathrm{NaOH} \mathrm{L}{ }^{-1}$. O P disponível e o $\mathrm{K}$ trocável foram obtidos com solução de extrato de Mehlich $1\left(0,05 \mathrm{~mol} \mathrm{HCl} \mathrm{L}{ }^{-1}+0,0125 \mathrm{~mol}\right.$ de $\left.\mathrm{H} 2 \mathrm{SO} 4 \mathrm{~L}^{-1}\right)$ e analisados por colorimetria e fotometria de chama, respectivamente. $\mathrm{O} \mathrm{H}+\mathrm{Al}$ foram extraídos com $0,5 \mathrm{~mol}$ de $\mathrm{CH} 3 \mathrm{COO} 2 \mathrm{Ca} \mathrm{L}^{-1}$ e determinados por titulação com solução de 0,025 mol de $\mathrm{NaOH} \mathrm{L}^{-1}$ e pH em água na proporção de 1:2,5 (solo: água).

Foram realizadas análises de variância (ANOVA) e teste de Tukey $(\alpha=0,05)$ para comparação de médias, utilizando-se o software Statistical Analysis System (SAS), versão 8.0. Os resultados do tempo de indução e recuperação foram analisados por regressão não- linear para a função com melhor ajuste dos dados.

\section{Resultados e Discussão}

A análise de sedimentos está sumarizada na Tabela $1 . \mathrm{O} \mathrm{pH}$ médio foi significativamente maior nos sedimentos que receberam calagem, adubação e peixes (T1), seguido dos tratamentos T2 (viveiros secos com calagem e sem peixe) e T3 
(tanques sem preparo e com peixes), respectivamente. $\mathrm{O}$ carbono orgânico e $\mathrm{Mg}^{+2}$ e $\mathrm{Al}^{+3}$ trocáveis foram superiores ( $\mathrm{p}<0,05$ ) em sedimentos de T1, em comparação com T2 e T3, que apresentaram valores intermédios e inferiores (p<0,05), respectivamente. A quantidade de matéria orgânica e K trocável foram maiores (p<0,05) em T1 e T3 quando comparado ao T2. O P disponível e Ca trocável foram significativamente maiores nos solos sem preparo calagem $(\mathrm{T} 3)$ e inferiores $(\mathrm{p}<0,05)$ nos tratamentos que receberam calagem ( $\mathrm{T} 1$ e $\mathrm{T} 2)$. O $\mathrm{H}+\mathrm{Al}$ trocável foi maior $(\mathrm{p}<0,05)$ em $\mathrm{T} 3$, seguida por $\mathrm{T} 1$ e $\mathrm{T} 2$, respectivamente.

Não houve correlação entre o pH com K, Mg, Al, H+Al, material orgânico $(\mathrm{p}>0,05)$. Mas houve correlação $(\mathrm{p}<0,05)$ entre $\mathrm{pH}$ com $\mathrm{Na}^{+}, \mathrm{Ca}^{+2}$, carbono orgânico positivo) e P disponível (Figuras 1 e 2).

Tabela 1. Média (desvio padrão) de $\mathrm{pH}, \mathrm{C}$ orgânico, matéria orgânica, $\mathrm{P}$ disponível e $\mathrm{K}, \mathrm{Na}, \mathrm{Ca}, \mathrm{Mg} \mathrm{e} \mathrm{Al}$ trocáveis em sedimentos de viveiros que receberam calagem, adubação e peixes (T1), viveiros com calagem e sem peixes (T2) e viveiros sem nenhum preparo e com peixes (T3).

\begin{tabular}{lccc}
\hline Parâmetros & $\mathrm{T} 1$ & $\mathrm{~T} 2$ & $\mathrm{~T} 3$ \\
\hline $\mathrm{pH}$ & $6,02 \pm 0,34 \mathrm{a}$ & $5,3 \pm 0,38 \mathrm{~b}$ & $4,18 \pm 0,34 \mathrm{c}$ \\
$\mathrm{C}$ org $\left(\mathrm{g} \mathrm{kg}^{-1}\right)$ & $13,43 \pm 1,81 \mathrm{a}$ & $2,24 \pm 0,41 \mathrm{c}$ & $5,30 \pm 0,73 \mathrm{~b}$ \\
Mat. orgânica $\left(\mathrm{g} \mathrm{kg}^{-1}\right)$ & $21,22 \pm 3,48 \mathrm{a}$ & $4,51 \pm 0,80 \mathrm{~b}$ & $21,82 \pm 2,31 \mathrm{a}$ \\
$\mathrm{P}$ disp $\left(\mathrm{mg} \mathrm{dm}^{-3} \mathrm{TFSA}\right)$ & $7,50 \pm 0,88 \mathrm{a}$ & $4,07 \pm 0,85 \mathrm{~b}$ & $5,24 \pm 0,90 \mathrm{~b}$ \\
$\mathrm{~K}^{+}\left(\mathrm{cmol}_{\mathrm{c}} \mathrm{dm}^{3}\right)$ & $0,34 \pm 0,05 \mathrm{a}$ & $0,23 \pm 0,05 \mathrm{~b}$ & $0,34 \pm 0,04 \mathrm{a}$ \\
$\mathrm{Na}^{+}\left(\mathrm{cmol}_{\mathrm{c}} \mathrm{dm}^{3}\right)$ & $0,27 \pm 0,06 \mathrm{ab}$ & $0,24 \pm 0,06 \mathrm{~b}$ & $0,39 \pm 0,08 \mathrm{a}$ \\
$\mathrm{Ca}^{+2}\left(\mathrm{cmol}_{\mathrm{c}} \mathrm{dm}^{3}\right)$ & $9,50 \pm 0,77 \mathrm{a}$ & $6,50 \pm 0,31 \mathrm{~b}$ & $6,48 \pm 0,61 \mathrm{~b}$ \\
$\mathrm{Mg}^{+2}\left(\mathrm{cmol}_{\mathrm{c}} \mathrm{dm}^{3}\right)$ & $4,32 \pm 0,49 \mathrm{a}$ & $2,58 \pm 0,12 \mathrm{c}$ & $3,64 \pm 0,15 \mathrm{~b}$ \\
$\mathrm{Al}^{+3}\left(\mathrm{cmol}_{\mathrm{c}} \mathrm{dm}^{3}\right)$ & $1,31 \pm 0,85 \mathrm{a}$ & $0,30 \pm 0,06 \mathrm{c}$ & $0,90 \pm 0,19 \mathrm{~b}$ \\
$\mathrm{H}+\mathrm{Al}\left(\mathrm{cmol}_{\mathrm{c}} \mathrm{dm}^{3}\right)$ & $5,58 \pm 0,62 \mathrm{ab}$ & $4,32 \pm 0,23 \mathrm{~b}$ & $6,59 \pm 0,66 \mathrm{a}$ \\
\hline
\end{tabular}

A média seguida da mesma letra na horizontal não difere estatisticamente pelo teste de Tukey $(\mathrm{p}<0,05)$. Fonte: Autores.

A camada inferior do sedimento dos viveiros de piscicultura é tão importante quanto a qualidade da água em considerações da estrutura e função desses viveiros (Boyd, 1990; Boyd et al., 2007; Olah, 1980). Partículas orgânicas e inorgânicas da água precipitam até os solos de viveiros de piscicultura, podendo ocorrer uma variedade de plantas, animais e microrganismos nesse microecossistema, onde há uma troca de substâncias entre o solo e a água. Na maioria dos casos são aplicados a calagem e fertilizantes durante a preparação dos viveiros de piscicultura, para melhorar a qualidade da água e a produção de biomas no ambiente aquático (Sonnenholzner \& Boyd, 2000). Neste estudo foi caracterizado os sedimentos de viveiros em estuários amazônicos, avaliando a preparação dos viveiro por meio de fertilização durante a piscicultura neotropical. 
Figura 1. Equação de regressão para a relação entre $\mathrm{pH}$ e íons trocáveis em sedimentos de piscicultura em estuários amazônicos. A: relação entre $\mathrm{pH}$ e $\mathrm{Na}$ trocável. B: relação entre $\mathrm{pH}$ e $\mathrm{Mg}$ trocável, C: relação entre o pH e Ca trocável, D: relação entre pH e K trocável, E: relação entre pH e Al trocável, F: relação entre o pH e H+Al trocável.
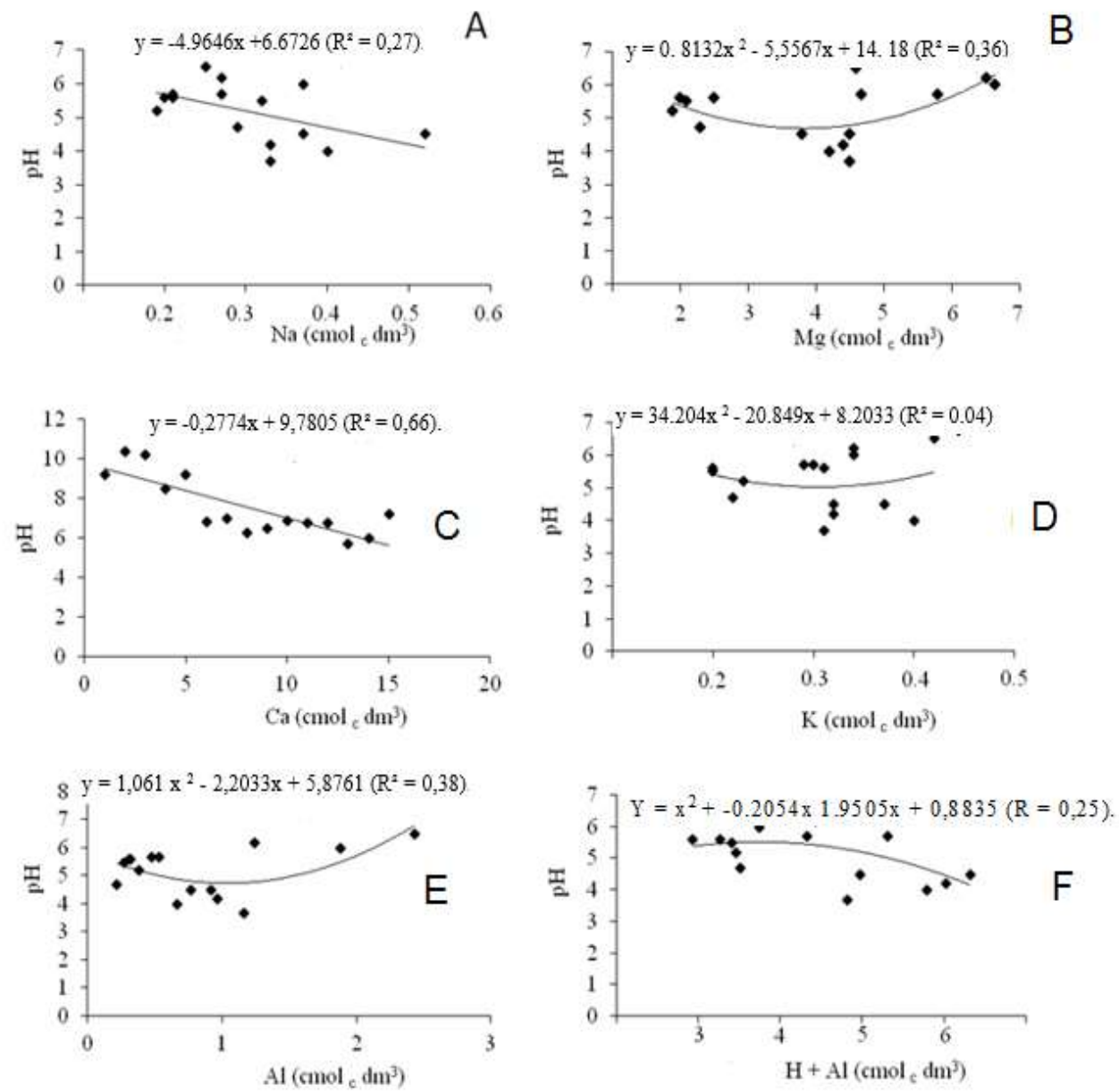

Fonte: Autores.

Sistemas semi-intensivos de piscicultura baseiam-se na calagem, secagem e adubação (Tacon; Silva, 1997). Nesses sistemas há interações complexas entre organismos alimentares naturais, suprimento alimentar endógeno e a prática de alimentação suplementar. Nesta pesquisa, os viveiros com calcário, fertilizantes e peixes (T1) promoveram elevada quantidade de matéria orgânica (carbono orgânico) e de fósforo disponível, principalmente devido ao uso de rações, para explicar o aumento de matéria orgânica (MO), e de adubo fosfatado para o aumento de $\mathrm{P}$, indicando o alto efeito residual do $\mathrm{P}$ nos sedimentos de tanque de piscicultura, o que foi confirmada por Tepe e Boyd (2002). Em geral, as concentrações de carbono, nitrogênio e fósforo aumentam em solos de viveiros ao longo do tempo, caracterizado pela dieta fornecida durante a criação de peixes. A relação entre carbono e nitrogênio $(\mathrm{C}: \mathrm{N})$, influencia na atividade microbiana, ou o sedimento pode adsorver ou liberar fósforo controlando as interações água-solo de acordo com a idade desse viveiro (Queiroz; Boeira, 2006; Boyd; Queiroz, 2014; Prihutomo et al., 2016).

Steeby et al. (2004) mostraram que carbono orgânico em sedimentos de viveiros com bagres de canal no Mississipi (EUA) variou entre 0,76 a 3,43\%, com média de 1,66\%. Não houve correlação entre carbono orgânico e idade dos tanques ou aumento da produção de peixe. Enquanto, Tepe e Boyd (2002) observaram o acúmulo de carbono, nitrogênio e fósforo em viveiros de aquicultura nos Estados Unidos. No entanto, Tian e Niu (2015) relatam que se deve ter o cuidado com a provável 
acidificação do sedimento como resultado da deposição de nitrogênio via fertilização nitrogenada, principalmente na forma de $\mathrm{N}$ amoniacal. A acidificação do solo induzida pelo $\mathrm{N}$ tem sido uma ameaça significativa à diversidade de espécies e ao funcionamento do ecossistema terrestre (Chen et al., 2013). Beveridge (2004) e Pillay (2004) mostraram que a piscicultura em tanques-redes não pode utilizar 30\% deste nutriente em rações formuladas com risco do aumento da matéria orgânica em rios. É interessante lembrar que para uma piscicultura sustentável, a produção e o meio ambiente devem estar em homeostase.

Figura 2. Equação de regressão para a relação entre pH e matéria orgânica (A), carbono orgânico (B) e P disponível (C) em sedimentos em viveiros de piscicultura em estuários amazônicos. A: relação entre $\mathrm{pH}$ e material orgânico, $\mathrm{y}=-0,0161 \mathrm{x}+$ 5,4285 $\left(\mathrm{R}^{2}=0,03\right)$. B: relação entre pH e carbono orgânico, $y=0,02 x 2-0,2502 x+5,4775\left(\mathrm{R}^{2}=0,42\right)$. C: relação entre pH e P disponível, y =0,116x $2-1,0586 x+7,167\left(R^{2}=0,47\right)$.
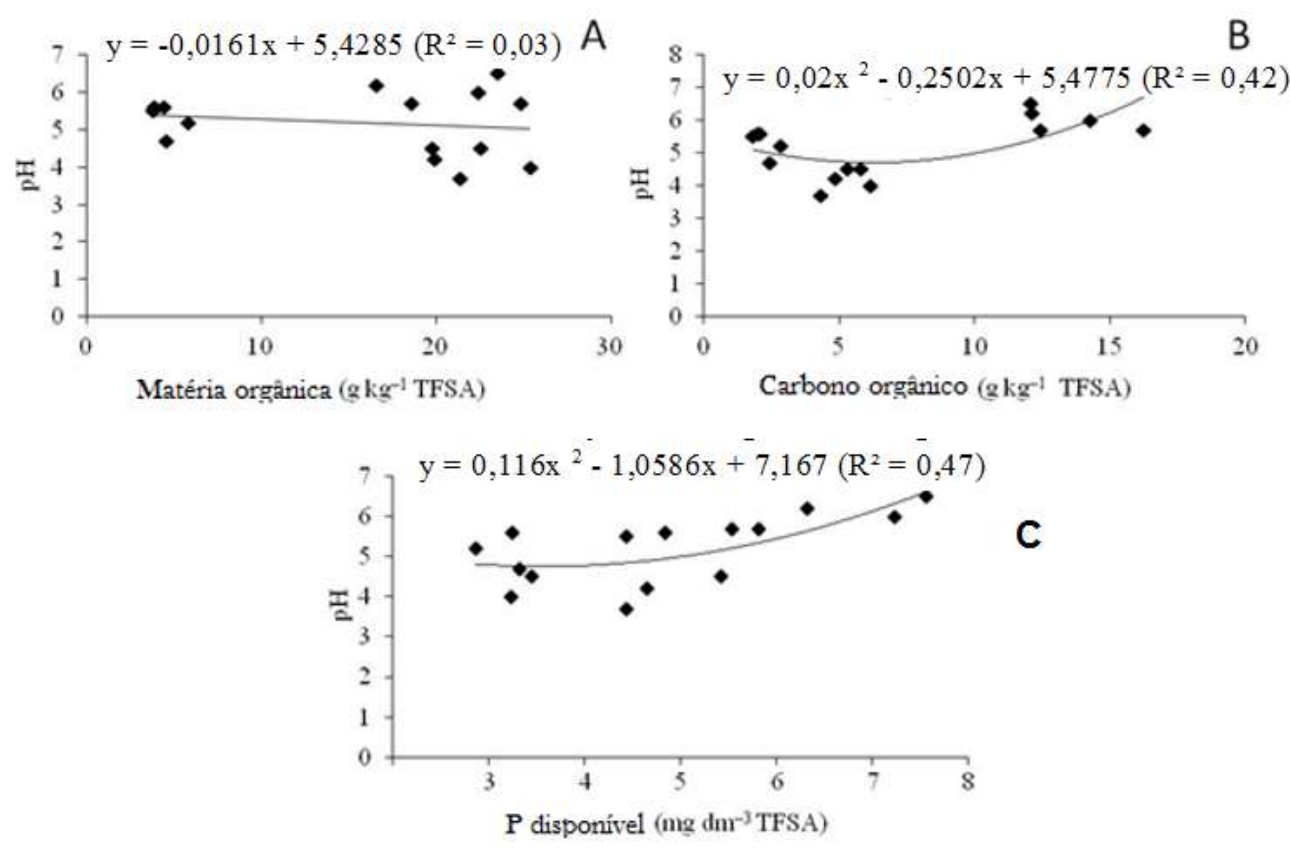

Fonte: Autores.

A integração agricultura-aquicultura tem sido bastante discutida como uma alternativa de aumentar a eficiência do uso da terra em virtude do aumento do crescimento populacional, a degradação ambiental e escassez de terra e água (Barg et al., 2000). O excesso de matéria orgânica produzida pela piscicultura pode ser utilizada como fertilizante natural na cultura do açaí, Euterpe oleracea na Amazônia. A calagem funciona principalmente para elevar o pH, a alcalinidade e a disponibilidade de carbono nas águas do viveiro, assim como para aumentar o pH dos sedimentos e melhorar a solubilidade do fósforo e a decomposição da matéria orgânica (Boyd, 1997).

A quantidade incrementada de matéria orgânica e C no sedimento do viveiro, no tratamento T2 (água com calagem sem peixes), pode estar relacionada ao material orgânico contido do próprio sistema e à taxa de decomposição por bactérias anaeróbias. Este evento foi confirmado por pH ácido encontrado no sedimento. A adoção de boas práticas de manejo adequadas para determinado tipo de corpo hídrico deve ser aplicada na aquicultura (Namdev, 2015; Yang et al., 2017). Cranwell (1976) mostrou que material orgânico em sedimento lacustre teve fonte alóctone resultante de produção primária e alóctone de ecossistema terrestre, ou seja, lixiviação. Além disso, o volume de água que entra nas várzeas pode promover a dissolução de C, N e P, diminuindo a deposição de sedimentos (Tepe \& Boyd, 2002).

Nos viveiros sem calagem e sem fertilização (T3) aumentaram o P disponível no solo, provavelmente devido a 
riqueza dos solos de várzea (Sioli, 1991; Lima; Tourinho, 1994; Brazão et al., 2018). Além disso, a concentração de carbono orgânico também aumentou como resultado da baixa decomposição microbiana. As concentrações de equilíbrio de dióxido de carbono são baixas em águas de baixa alcalinidade, e o dióxido de carbono da respiração microbiana se difundirá no ar quando as concentrações de dióxido de carbono excederem o equilíbrio. Quando o carbonato de cálcio é aplicado em viveiros de piscicultura nos quais grandes quantidades de dióxido de carbono são liberadas por decomposição, sua solubilidade será aumentada (Somridhivej \& Boyd, 2017). Além disso, os materiais cálcicos provavelmente se dissolvem mal em águas salgadas. No entanto, os aquicultores em todo o mundo aplicam grandes quantidades de calcário agrícola e calcário em viveiros (Sá \& Boyd, 2016). Boyd et al. (2016) sugeriram que os materiais cálcicos adicionados aos viveiros muitas vezes não se dissolvem, porque a água já está saturada com $\mathrm{CaCO}_{3}$.

O processo de calagem foi crítico para os sedimentos nos estuários da Amazônia e o baixo pH foi encontrado em viveiros sem tratamento. Acidificação de estuários por subprodutos da aquicultura, ou seja, ácidos orgânicos, compostos orgânicos reduzidos de enxofre, manganês e sulfetos reduzidos, pode ocorrer em todo o mundo como resultado da ação humana (Sammut et al., 1995; Avnimelech \& Ritvo, 2003; MacDonald et al., 2004; Yang et al., 2017). A aquicultura é uma das práticas antropogênicas que mal manejadas podem interferir no ecossistema aquático (Thornberg et al., 2014; Yang et al., 2017) e, dessa forma, os procedimentos corretos em viveiros escavados poderiam melhorar a condição de água e sedimentos, evitando a acidificação. Nesse estudo, a calagem foi um método eficiente para evitar que o pH do solo diminuísse em viveiros de criação de peixes em ambiente amazônico.

Foram encontradas correlações entre o $\mathrm{pH}$ e $\mathrm{Na}^{+}, \mathrm{Ca}^{2+}$, carbono orgânico e $\mathrm{P}$ disponível, com correlação negativa entre $\mathrm{pH}$ e Na. Apenas o Na trocável apresentou resposta positiva em relação ao $\mathrm{pH}$, provavelmente devido à baixa capacidade tampão em solo sem calagem. Li et al. (2017) mostraram correlações negativas muito significativas entre o pH do solo e o teor de sal no solo, assim como o pH e a maioria dos íons solúveis no solo. Além disso, o aumento da concentração de dióxido de carbono atmosférico (CO2) está aumentando a solubilidade do calcário, silicato de cálcio e feldspatos, resultando em maior concentração de alcalinidade total em águas interiores (Hartman et al., 2016; Somridhivej et al., 2017). Nos estuários da Amazônia havia ainda a água salgada para melhorar as concentrações de íons Na e o procedimento sem calcário pode aumentar a inativação do sedimento.

A concentração pouco elevada de $\mathrm{Al}^{+3}$ embora bem menor ao que foi encontrado por Colares (2007) em solos alagados, sugere a citação de Anghinoni e Bissani (2004) de que os corretivos adicionados no solo foram insuficientes, assim como a adição de $\mathrm{K}$ seria ideal para a neutralização do $\mathrm{Al}^{+3}$. Talvez a adição do fertilizante nitrato de potássio fosse providencial para a inibição do $\mathrm{Al}^{+3}$. A calagem é basicamente um meio de neutralizar a acidez do solo e, nos estuários da Amazônia é necessário adequar o sedimento durante a produção de peixes. O manejo correto dos viveiros de solos em estuários pode minimizar os problemas ambientais causados pelas modificações antrópicas impostas.

\section{Conclusão}

Os solos de viveiros de piscicultura em várzeas de estuário amazônico são susceptíveis à calagem, e manejo de fertilizantes.

Portanto, o manejo correto na preparação dos viveiros em várzeas estuarinas pode melhorar a qualidade do solo para piscicultura nesse tipo de agroecossistema.

\section{Referências}

Anghinoni, I., \& Bissani, C. A. (2004). Correção da acidez do solo e materiais utilizados. IN: Bissani, C.A., Gianello, C., Tedesco, M. J., Camargo, F. A. O. C. Fetilidade dos solos e manejo da adubação de culturas. Porto alegre, p. 153-165. 
Aires, J. M. (1993). C Como matas de várzea do Mamirauá. MCT-CNPq-Programa do Trópico Úmido. Sociedade Civil do Mamirauá, Brasil, 123p.

Arauco, L. R. R, Cruz, C, \& Machado Neto, J. G. (2005). Efeitos da sedimentação na toxicidade aguda do sulfato de cobre e triclorfon para três espécies de Daphnia. Pesticidas R.ecotoxicol. e meio ambiente, (15): 55-64.

Avnimelech, Y., \& Ritvo, G. (2003). Camarões e viveiros de peixes: processos e gestão. Aquicultura, 220: 549-567, 2003.

Barg, U., Bartley, D., Kapetsky, J., Pedini, M., Satia, B., Wijkstrom, U., \& Willmann, R. (2000). Gestão integrada de recursos para a produção sustentável de peixes no interior. FAO Aquaculture Newslettr, 23, 4-8.

Bastos, T. X. (1972). O estado atual dos conhecimentos, das condições climáticas da Amazônia brasileira. Instituto de Pesquisa Agropecuária da Amazônia Brasileira. Instituto de Pesquisa Agropecuária do Norte - IPEAN, Belém. Boletim Técnico. 54: 68-112.

Beveridge, M. C. M. (2004). Cage aquicultura. (3a. ed.), Blackwell Publishing, 368p.

Boyd, C. E. (1995). Solo inferior, sedimento e aquicultura em viveiros. Chapman e Hall.

Boyd, C. E. (1982). Gestão da qualidade da água para a piscicultura. Amesterdão Elsevier Scientific Publishing, p. 318.

Boyd, C. E. (1990). Qualidade da água em viveiros para a aquicultura. Birmingham Publishing Co., Primeira Impressão, Alabama, p. 482.

Boyd, C. E., \& Queiroz, J. F. (2014). O papel e manejo de solos de fundo em viveiros de aqüicultura. Infofish International, 2, $22-28$.

Boyd, C. E., Tucker, C. S., \& Somridhivej, B. (2016). Alcalinidade e dureza: conceitos críticos mas ilusórios na aquicultura. J. World Aquac. Soc. 47, 6-41.

Boyd, C. E., Tucker, C. S., Mcnevin, A., Bostick, K., \& Clay, J. (2007). Indicadores de eficiência de uso de recursos e desempenho ambiental em peixes e aquacultura de crustáceos. Avaliações no Fisheries Scicence, 15: 327-360.

Burton, G. A., \& Lamdrum, P. (2004). Toxicidade de sedimentos. Em: Rocks, G, V., Middleton, M. J, Church, M, Corigilo, L. A (Eds.) Enciclopédia de sedimentos e sedimentares. Dordrecht: Kluwer Academic, 747-771.

Carvalho Filho, J. A produção agrícola de 2017. 4,94\% em 2016, enquanto a carcinicultura, teve uma queda de 25,39\%. Panorama da Aquicultura.

Chelossi, E., Vezzulli, L, Milano, A., Branzoni, M, Fabiano, M., Riccardi, G, \& Banat, I. M. (2003). Resistência antibiótica de bactérias bênticas em fazendas de peixes e controle de sedimentos do Mediterrâneo Ocidental. Aquicultura, p.1 -15.

Chen, H., Liu, C., Xu, X. R., Liu, S. S., Zhou, G. J., Sun, K. F., Zhao, J. L., \& Ying, G. G. (2014). Antibióticos em fazendas típicas de aquicultura marinha em torno da ilha de Hailing, no sul da China: Ocorrência, bioacumulação e exposição alimentar humana. Boletim de Poluição Marinha.

Colares, M. T. (2007). Efeito da calagem em solos de açude com diferentes valores de CTC sobre a alcalinidade e a dureza da água. Ver. Bras. Agrociência. Pelotas, 13(4), 491-494.

Cranwell, P. A. Compostos orgânicos como indicadores de entrada alóctone e autóctones em sedimentos de viveiros. Em: Interações entre sedimentos e água doce. Amsterdam,

EMBRAPA - Empresa Brasileira de Pesquisa Agropecuária. 1997a. Centro Nacional de Pesquisa de Solos, Manual de Métodos de Análise de Solos. (2a ed.) Rio de Janeiro, Embrapa, 1997. 212.p.

EMBRAPA - Empresa Brasileira de Pesquisa Agropecuária. 1997b. Centro Nacional de Pesquisa de Solos, Manual de Métodos de Análise de Solos. (2a ed.) Rio de Janeiro, Embrapa, 1997. 23.p.

Espectroscopia de absorção de Slavin, W. Atomico. Interciência, 1968. 307p.

FAO - Organização para a Alimentação e Agricultura das Nações Unidas. O estado das pescas e aquacultura mundiais contribuindo para a segurança alimentar e nutricional para todos. Roma. ISSN 1020-5489. 200p.

Grigorakis, K., \& Rigos, G. (2011). Efeitos da aquacultura no ambiente e bem-estar público - o caso da maricultura mediterrânica. Chemosphere, 85 (6), 899919, 2011.

Gross, A., Boyd, C. E., \& Wood, C. W. (1999). Volatilização de amônia de viveiros de água doce. Jornal de Qualidade Ambiental, $28:$ 793-797, 1999.

Gross, A., Boyd, C. E, \& Wood, C. W. (2000). Transformações de nitrogênio e equilíbrio em viveiros de bagres de canal. Engenharia Aquícola, 24: 1-14.

Guimarães, G. A, Bastos, J. B, \& Lopes, E. C. (1970). Métodos de análise física, química e instrumental de solos. Belém, IPEAN, 108 p.

Hartman, P., Kutý, M., Hlaváč, D., Regenda, J., \& Vejsada, P. (2016). Teor de cálcio no sedimento da viveiro e seu efeito na capacidade neutralizante da produção de água e peixe. Aquicultura internacional, 24, 1747-1754.

Jackson, M.L (1962). Análise química do solo. Englewood Clifs: Prentice-Hall.

Li, Q, Xi, M, Wang, Q, Kong, F., \& Li, Y. (2017). Caracterização da salinização do solo em área estuarina típica da Baía de Jiaozhou, China. Física e Química da Terra.

Lima, R. R., \& Tourinho, M. M. (1994). Várzeas da Amazônia Brasileira: principais características e possibilidades agropecuárias. Belém: FCAP. 20 p. 
Lu, X.M, Dang, Z, \& Yang, C. (2009). Investigação preliminar de cloranfenicol em peixes, água e sedimentos da viveiro de aquacultura de água doce. Int. J. Environ. Sci. Tech., 6 (4), 597-604.

Macdonald, B.C.T, Smith, J, Keene, Af, Tunks, M, Kinsela, A., \& White, I. (2004). Impactos do escoamento de solos sulfurosos na química dos sedimentos em um viveiro e estuarino. Ciência do Meio Ambiente Total, 329: 115-130.

Meijer, L.E, \& Avnimelech, Y. (1999). Sobre o uso de microeletrodos em sedimentos de viveiros de peixes. Engenharia Aquícola, 21, 71-83.

MPA - Ministério da Pesca e Aquicultura - Boletim Estatístico da Pesca e Aquicultura - 2011. Brasília: Ministério da Pesca e Aquicultura, 60p. http://www.mpa.gov.br/images/Docs/Informacoes_e_Esta tisticas / Boletim\% 20MPA\% 202011FINAL.pdf .

Namdev, U. (2015). Práticas de aquicultura em solos de fundo de viveiro ácida na região NE da Índia. Revista Internacional de Tecnologia de Engenharia e Ciências Aplicadas. 1, 1-8.

Olah, J. (1980). Quantificação estrutural e funcional em uma série de viveiros rasos hipertróficos húngaros. Páginas 191-202. In: J. Barica e LR Mur, editores. Desenvolvimento em Hydrobiology, volume 2. Dr. W. Junk bv Pubs, The Hague, Holanda.

Ostrensky, A., \& Boeger, W. (1998). Piscicultura: fundamentos e técnicas de manejo. In: Ostrensky, A., Boeger, W. (Eds.). Guaíba: Agropecuária, 211p.

Peterson, E. L (1999). Esforço de cisalhamento bentônico e condição de sedimento. Engenharia tural Aquacul, 21 (2): 85-111.

Peterson, E. L (2000). Observação da hidrodinâmica da viveiro. Engenharia Aquícola, 21 (4), 247-269.

Peterson, E. L, Harris, J. A., \& Wadhwa, L. C. (2000). Processos de dinâmica de modelagem de CFD. Engenharia Aquícola, 23 (1- 3 ): 61-93.

Peterson, E. L, Wadhwa, L.C, \& Harris, J.A (2001). Arragment de aeradores em uma viveiro de cultivo de camarão intensivo que tem uma forma retangular. Engenharia Aquícola, 25 (1): 51-65.

Peterson, E. L. (1999). O efeito dos arejadores na tensão de cisalhamento bentônico em uma viveiro. Tese de doutorado (tese de doutorado). James Cook University of North Queensland Townsville, Austrália. Chapers no volume 1,291p.

Prihutomo, A., Hardanu, W., \& Triana, K. A. (2016). Uso do Índice de Qualidade de Solo Modificado para Determinação de Viveiros Estado da Qualidade do Solo Inferior da Área de Aquicultura BLUPPB Karawang West Java, Indonésia.

Queiroz, J. F., \& Boeira, R. C. (2006). Calagem e controle da acidez dos viveiros de aquicultura. Jaguariúna: Embrapa Meio Ambiente, 2006.8 p. il. (Embrapa Meio Ambiente. Técnica Circular, 14).

Queiroz, J. F, Boeira, R. C., \& Silveira, M. P. (2004). Coleta e Preparação de Amostras de sedimentos de viveiro s de aquicultura Jaguariúna: Embrapa Meio Ambiente, 5p. il. Embrapa Meio Ambiente. Comunicado Técnico, 17.

Rico, A., Satapornvanit, K., Haque, M. M., Min, J., Nguyen, P., Telfer, T., \& Van Den Brink, P. J. (2012). Uso de produtos químicos e biológicos na aquicultura asiática e seus potenciais riscos ambientais: uma revisão crítica. Rev. Aquacult, 4 (2), 75-93.

Sá, M. V. C., \& Boyd, C. E. (2016). Taxa de dissolução de carbonato de cálcio e hidróxido de cálcio em águas salinas e sua relevância para a aquicultura Aquicultura.

Sammut, J., Melville, M. D., Callinan, R. B., \& Fraser, G. C. (1995). Acidificação de estuários: impactos na Biota aquática da drenagem de solos sulfatados ácidos. Aust ralian Studies, 33 geográfica: 89-100.

Schmidt, A. J., Bruun, M. S., Dalsgaard, E. U., Pedersen, K., \& Larsen, J. L. (2000). Ocorrência de resistência antimicrobiana em bactérias patogênicas e ambientais associadas a quatro fazendas dinamarquesas de trutas arco-íris. Microbiologia aplicada e ambiental, 66, 4908-4915.

Silva, S. B. (1991). Análise química de solos. Belém, FCAP. Serviço de Documentação e Informação, 41 p (FCAP. Informe Didático, 11).

Sioli, H. (1991). Amazônia: Fundamenyos da ecologia da maior região de florestas tropicais. Vozes, 72 p.

Sonnenholzner, S., \& Boyd, C. E. (2000). Propriedades químicas e físicas do fundo do viveiro de camarão. Solos no Equador. Jornal da sociedade da aquacultura, 31: 358-375.

Steeby, J. A, Hargreaves, J. A., Tucker, C. S., \& Kingsbury, S. (2004). Acumulação, concentração de carbono orgânico e matéria seca de sedimentos em viveiros de bagres de canais comerciais. Engenheiro Aquacultural, 30: 115-126.

Tacon, A. G. J, \& Silva, S. S. D. (1997). Preparação de rações e estratégias de manejo de rações dentro de sistemas semi-intensivos de piscicultura nos trópicos. Aquicultura, 151, 379-404.

Tepe, Y, \& Boyd, C. E. (2002). Qualidade de sedimentos em viveiros de minnow bait Arkansas. Jornal da sociedade mundial de aquacultura, 33 (3): 221 232.

Thomas, G. W. (1982). A fração coloidal: assento da atividade química e física do solo: cátions intercambiáveis. Em: A natureza e propriedades dos solos. Ray R. Weil, Nyle C. Brady (edts.) Décima Quinta Edição. Columbus: Pearson, 2016, 327-373.

Thunjai, T., Boyd, C. E., \& Boonyaratpalin, M. (2004). Qualidade do solo inferior em viveiros de tilápias de diferentes.

Tian, D., \& Niu, S. (2015). Uma análise global da acidificação do petróleo causada pela adição de nitrogênio. Environ Res. Lett. 10, 024019.

Vetori, L. (1969). Métodos de análise de solos. 24 p. (Boletim Técnico, 16). 
Research, Society and Development, v. 10, n. 1, e41710111815, 2021

(CC BY 4.0) | ISSN 2525-3409 | DOI: http://dx.doi.org/10.33448/rsd-v10i1.11815

Vogel, A. (1981). Análise inorgânica quantitativa. (4a ed.), 690p.

Yang, P., Lai, D., Jin, B., Bastviken, D., Tan, L., \& Tong, C. (2017). Dinâmica dos nutrientes dissolvidos nas viveiros de camarão de aquacultura do estuário do rio Mín, China: Concentrações, fluxos e cargas ambientais. Ciência do Meio Ambiente Total. 603-604: $256-267$.

Zhao, Q., Bai, J., \& Lu, Q. (2016). Efeitos da salinidade na dinâmica do carbono do solo em áreas úmidas costeiras degradadas: implicações na restauração de áreas úmidas. Phys. Chem. Terra. 2016. 